\title{
Understanding Kinaesthetic Creativity in Dance
}

\author{
Stacy Hsueh $^{1} \quad$ Sarah Fdili Alaoui $^{1} \quad$ Wendy E. Mackay ${ }^{1}$ \\ ${ }^{1}$ Univ. Paris-Sud, CNRS, \\ Inria, Université Paris-Saclay \\ Orsay, France \\ hsueh@lri.fr, saralaoui@lri.fr, mackay@lri.fr
}

\begin{abstract}
Kinaesthetic creativity refers to the body's ability to generate alternate futures in activities such as role-playing in participatory design workshops. This has relevance not only to the design of methods for inspiring creativity but also to the design of systems that promote engaging experiences via bodily interaction. This paper probes this creative process by studying how dancers interact with technology to generate ideas. We developed a series of parameterized interactive visuals and asked dance practitioners to use them in generating movement materials. From our study, we define a taxonomy that comprises different relationships and movement responses dancers form with the visuals. Against this taxonomy, we describe six types of interaction patterns and demonstrate how dance creativity is driven by the ability to shift between these patterns. We then propose a set of interaction design qualities to support kinaesthetic creativity.
\end{abstract}

\section{CCS CONCEPTS} -Human-centered computing $\rightarrow$ HCI theory, concepts
and models;

\section{KEYWORDS}

Kinaesthetic creativity; movement generation; dance

\section{ACM Reference Format:}

Stacy Hsueh, Sarah Fdili Alaoui, and Wendy E. Mackay. 2019. Understanding Kinaesthetic Creativity in Dance. In CHI Conference on Human Factors in Computing Systems Proceedings (CHI 2019), May 4-9, 2019, Glasgow, Scotland UK. ACM, New York, NY, USA, 12 pages. https://doi.org/10.1145/3290605.3300741

CHI 2019, May 4-9, 2019, Glasgow, Scotland UK (c) 2019 Association for Computing Machinery.

This is the author's version of the work. It is posted here for your personal use. Not for redistribution. The definitive Version of Record was published in CHI Conference on Human Factors in Computing Systems Proceedings (CHI 2019), May 4-9, 2019, Glasgow, Scotland UK, https://doi.org/10.1145/3290605. 3300741.

\section{INTRODUCTION}

There is ongoing interest in HCI in designing for the experiential, sensorial body, which is influenced by the phenomenological perspective that the human body, in addition to being an object in the world, also exists as a lived body, with feelings, sensations, and emotions [44]. Researchers have designed both systems [22] and methods [23, 33] to engage with the lived body and to disrupt its movement habits, questioning what Sheets-Johnstone calls "making the familiar strange" [46]. These systems and methods share a common goal of supporting the body in accessing the felt dimension in order to cultivate bodily awareness or to design for movement-based interaction.

This paper builds on these works to explore the emergent and dynamic relations between the moving body and interactive technology during creative processes such as movement ideation: What are the mechanisms by which technology impacts the body's ability to generate creative materials? In addition to the different ways users interact with technology, how do these interactions evolve over time? What concepts and frameworks are needed to understand these processes? We focus on the embodied creative process related to the notion of "kinaesthetic creativity", coined by Svanaes [52], to describe the body's ability to enact alternate future possibilities via movement. Understanding this process can help us envision the qualities movement-based systems should adopt to better support it.

We ground our investigation in an empirical study of technology-mediated movement improvisation in dance. In order to probe this process, we developed a series of interactive visualizations and asked dance practitioners to explore with them to generate movements. Based on the study, we develop a taxonomy consisting of different relationships and movement responses dancers form with the visuals. We use this as basis to identify six interaction modes with the visuals dancers can enter into. Finally, we discuss the three primary strategies dancers use to produce creative materials by shifting between these modes. We then provide implications for the design of technologies that support creative movement generation and exploration. 


\section{RELATED WORK}

\section{$\mathrm{HCl}$ and the moving body}

As typified by the third wave of HCI [1], there is a move toward designing for the body and embodied experience $[16,22,45]$. In particular, researchers propose various design approaches to support movement generation and ideation, through designing for kinaesthetic awareness [16], estrangement [5, 58], and somaesthetic interactions [22].

Existing movement-based practices work toward breaking habits: those that humans form when understanding the world, training and learning to act on it. These habits support humans to cope with everyday life but sometimes disservice them by becoming tacit and difficult to change. Feldenkrais body-based method aims to disrupt movers' habits and has been applied in HCI to allow users to gain movement awareness [32]. Philosophies such as somaesthetics [47], inspired both by Feldenkrais and by phenomenological considerations of human movement in perception [21], have influenced a generation of HCI methods [24, 31, 57]. Amongst them are somaesthetic appreciation design proposed by Hook et al. [22], move to get moved [23], move to design [57], moving and making strange [33], and embodied sketching [36].

\section{Studying dancers and choreographers}

Multiple design approaches have emerged in HCI to support dance-making, favoring improvisation [33], annotation [4], documentation [7], or visualization [19, 37]. Alternatively, theories in modern dance [38] and studies with expert dancers and choreographers have opened new ways to formalize the body in HCI and to explore new paradigms for interaction $[11,12]$. The most common system that comes from dance, and that is used to articulate movement during interaction, is the Laban Movement Analysis. Maranan et al. developed a prototype composed of a wearable accelerometer system and software interface called "EffortDetect" to recognize Laban Efforts [35]. Fdili Alaoui et al. explored the Laban system for Effort recognition using multimodal interactions [13] and vocalizing these Efforts for dancers to explore their movement qualities in an improvisational dance workshop [17]. These principles were also applied to movement-based games [39, 40, 56].

Interactive arts, without formalizing it, build on intuitive and aesthetic knowledge of the body to propose immersive interactive experiences engaging various aspects of movement $[30,49,53]$. Many of these works promote creative uses of the body and propose visualizations as means to give feedback to participants on different representations of their movement. We build upon these previous works in movement-based interactions and existing ways of aesthetically visualizing the body to propose a set of visualizations that probe user's kinaesthetic creativity.

\section{Kinaesthetic creativity for movement-based interaction}

Kinaesthesia, or proprioception, is the sense of one's own movement execution. It operates at multiple levels of motor control such as muscular effort and position in space [55]. Proprioception has been applied in HCI, by using movement sensing with electrical muscle stimulation, to support kinaesthetic awareness and movement execution [34]. Fogtmann et al. propose kinaesthetic design with three design themes: "kinaesthetic development" that allows to acquire and improve bodily skills, "kinaesthetic means" that allows to reach another goal, and "kinaesthetic disorder" that allows for a transformation of the kinaesthetic experience[15]. This was further developed by [16], presenting kinaesthetic awareness as a critical value in embodied interaction design.

Kinaesthetic creativity refers to the general concern of "the active use of the body through abstract movements to explore possible futures" [52]. Abstract movements are movements performed as part of an isolated event (e.g. being asked to put one foot before another) rather than as part of everyday life (e.g. walking) [52]. Svanaes provides an account of their emergence in role-playing scenarios where participants diverge from enacted everyday movements with a device into imagined new interactions with it. This carving out of an imaginary zone for reflection within the everyday characterizes kinaesthetic creativity, of which we argue, in addition to being used to access the "feel" dimension of artifacts, can be designed for. We link this concern of "enacting alternate futures" to the goal of breaking movement habits and supporting movement ideation of end users.

We aim in this paper at probing the mechanisms of this creative process by letting users interact with a set of visual artifacts that are designed to represent the body and the movement. By doing so, we hope to understand the strategies dancers use to generate creative movement materials.

\section{DESIGNING THE CHOREOPROBE}

We designed a Kinect-based visualization system that tracks movement contours and dynamics. We deployed it in workshops as a technology probe [25] to help us understand how dancers ideate using visual artifacts.

To explore different options for the visualizations, we compiled movement visualization artworks by professional artists including Golan Levin [30], OpenEnded Group [42], Universal Everything [10], and onformative [41]. We categorize the visualizations as follows: visualizing movement structure $^{1}$, movement dynamics ${ }^{2}$, and body form ${ }^{3}$. Our probe consists of a set of visualizations that covers this design space.

\footnotetext{
${ }^{1}$ Such as Ghostcatching by OpenEnded Group

${ }^{2}$ Such as Unnamed Soundsculpture by onformative

${ }^{3}$ Such as Isosurface by Golan Levin
} 
We chose to use particle systems as the basis for our visualizations given their capacity to express dance movement qualities [11]. They are a common technique in computer graphics that uses a large number of independent objects to simulate different effects and behaviors.

\section{Visualization vignettes}

We created parameterized, interactive visuals, as seen in Figure 1, which we envisioned as "visualization vignettes".

Particles. We implemented a simple particle system and defined a set of rules the particles follow over time. Particles are emitted at every frame on the user contour tracked by the Kinect. A gravity force is applied to all the particles inside the contour. The direction of the force is determined by a vector across a $2 \mathrm{D}$ plane, so that they can be flying upwards or falling downwards. The controllable parameters are: direction of falling particles along the $\mathrm{x}$ and $\mathrm{y}$ axes, the particle size, and the particle lifetime.

Springs. We created a particle-based spring system within which particles get spawned and attached to the surface of the tracked contour. The goal is to simulate strings sprouted around the body whose shapes range from flowing fur-like strands to abstract "lineforms". Each particle is assigned a repulsion force from other particles proportional to the speed of user movements (the faster the movement, the stronger the force). The particles then link up to form animated curved lines around the contour. The controllable parameters are: rigidity of the springs, their bounce, their length, and their shape.

Blobby form. In this visualization, to give the $2 \mathrm{D}$ contour a more life-like texture, we turn the detected contour into a triangulated mesh where the vertices are dynamically colored based on a distance function. The users can control the form's "blobbiness", or the level of fidelity to the detected contour shape. In other words, the more "blobby" it is, the more the visualized body morphs into a blob.

Fluid body. To simulate fluid flow, we determine the velocity field at each time step by solving Navier-Stokes equations which describe the evolution of the fluid under a variety of forces. We used a solver library in OpenFrameworks. The user can control the fluid's flow rate.

Trails. This visualization keeps a cache of traversed points in space and connects them to form spatial trails. The cache gets cleared after a specifiable amount of time. The user can control the duration of the trails.

\section{ENGAGING DANCE PRACTITIONERS}

We held three separate observation sessions with two choreographers and 15 dancers. The aim is to articulate through these experimentation how dance practitioners interact with and through visual artifacts to generate movement.

\section{Setup}

We ran each session in a local dance studio (except for Workshop 3 which took place in a general use facility for exhibitions, workshops, and recreation), which had a wall onto which to project the visuals. The Kinect was placed on a tripod. The dancers moved in the space facing the camera and the projection.

\section{Workshop 1: dance and choreography course}

Participants. A total of six dancers (five women, one man; ages 19-32) followed the course with a choreographer and took part in a final performance. They had between 5 to 16 years of dance experience.

Procedure. The interactive visuals were used over the course of a three-day class. The students had already choreographed movement materials, and the choreographers asked the dancers to adapt and integrate the visuals into the choreography for the final performance. Each class lasted approximately 4 hours. The workshop was followed by semi-structured interviews with the participants ranging from 30 to 90 minutes. We used a variation of Flanagan's Critical Incident Technique [14] to capture open-ended but detailed stories of how participants used live visuals to generate movement material.

\section{Workshop 2: improvisation session with dancers}

Participants. We recruited six dancers (five women, one man) through our personal network to participate in a single-day workshop. They have between 6 to 30 years of dance trainging, ranging from modern jazz to contemporary dance. They were not compensated for their participation. They were however invited for lunch before the workshop.

Procedure. The session lasted approximately one hour. We gave a presentation of each visualization and its parameters and asked the participants to generate movement materials inspired by the visuals. They took turns - one dancer would manipulate the parameters while the others explored the visuals. We kept the session open-ended and exploratory: the dancers could choose to interact with the visuals in solo, in pairs, or in any configuration of their choosing. They were not given strict directives as to how they should relate to the visuals. They were encouraged to interpret them as they wish. We used a think-aloud protocol, encouraging them to describe what they saw and felt at their convenience, sometimes during slow movements and sometimes during pauses between movements. After the exploration, we asked them to explain how they perceived the visuals and what movement they were able to generate, using the same interview technique as above. 

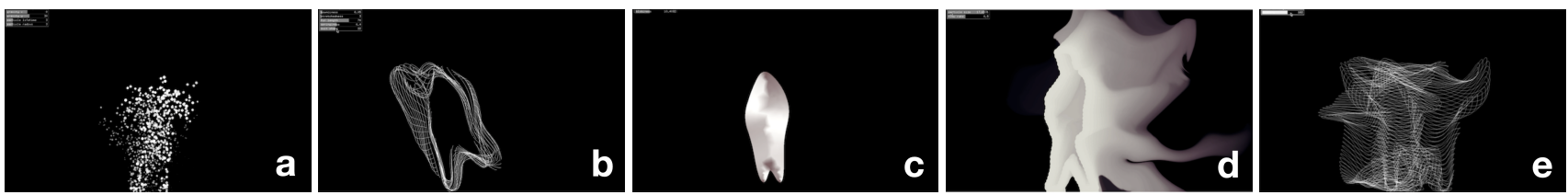

Figure 1. Visualization vignettes: (a) Particles (b) Springs (c) Blobby form (d) Fluid body (e) Trails

\section{Workshop 3: improvisation session with dancers and choreographers}

Participants. We recruited one choreographer and two dancers (all women) to participate in a single-day workshop. The choreographer has more than 10 years of experience in contemporary choreography and the dancers have around 5-6 years of training, one in modern jazz, and another in contemporary dance. The choreographer's collaborator also participated in the workshop as an observer; we refer to him henceforth as $\mathrm{P}$ (for participant).

Procedure. The session lasted approximately two hours. We gave a scripted presentation of the features of each visualization and asked the participants to perform the following tasks: generating movement materials and transforming movement materials. For the first task, the choreographer was asked to pick three visualizations as starting point for generating three movement sequences. The visualizations can be of different types, or of the same type but with different parameter settings. For the second task, the choreographer was asked to break dancers' movement patterns by manipulating parameters on the chosen visualization or to change to a wholly new visualization. We used the same think-aloud protocol as Workshop 2. After the tasks, we asked them to explain why they chose the specific visuals during movement generation. We also asked them to describe the changes they made to the visuals during transformation.

\section{Data collection}

We placed a camera on a tripod behind each participant to record video and audio of each session; we also took pictures, close-up videos, and written notes.

\section{Data analysis}

We transcribed and analyzed all of our notes, pictures, and videos using Thematic Analysis [2] in order to find common patterns across participants and to capture notable practices. We generated codes and themes inductively and deductively, using a bottom-up approach. We grouped codes into larger categories, focusing on dancers' relationships to the visuals, their movement behaviors, and their strategies for generating new movements. At each iteration, we checked back with transcribed data for consistency. Two authors did the analysis, and the third checked it. Acknowledging the inherently interpretive nature of qualitative analysis, we constructed a shared understanding during our analysis process by focusing on participants' own reflections on their experiences, corroborated with field notes and observation data. Each participant is given a code name based on their role in the workshop (dancer [D], choreographer [C], or participant [P]) and the workshop in which they participated (1,2, or 3). For example, dancer 4 from Workshop 2 is labeled D4W2 and choreographer from Workshop 3 is CW3.

\section{FINDINGS}

The observations and interviews unveiled a diverse set of interaction patterns dancers develop with the visuals. These patterns are not static; they are dynamically constituted and reproduced in an ongoing process during workshops.

We develop a taxonomy for this revealed set of interaction patterns. We place the different patterns along two dimensions. The first of the dimensions, "relationship to visuals", refers to the different ways dancers relate to visuals, as an instrument, a partner, or a medium. When employed as an instrument, the visuals are objects containing properties that can be mobilized during movement, helping dancers form a first-person relationship. For example, a dancer can use her visualized body as a brush, leaving traces on the virtual canvas via movements. The visuals can also serve as a dance partner with behaviors of their own, facilitating dancers' second-person relationship to them. Finally, the visuals can be used as a medium, mediating communication between people, thereby fostering a third-person relationship.

The second dimension, "movement types", refers to two kinds of movement behaviors that emerge as a result of the visuals configuring the dancers spatially and kinaesthetically: reactive and self-reflexive. In reactive movements, dancers move in response to constraints set by particular contexts, for example, the conic space delineated by Kinect's range of capture. In self-reflexive movements, dancers turn their attention from the external environment back to the movement itself: instead of conforming to situation-relative constraints, dancers place the primacy back on movement: making movements for movement's sake.

In the following sections, we discuss the different modes of interaction that result from crossing these two dimensions and provide illustrative examples from our interviews and 
observations. We then report the strategies dancers use to shift between different interaction patterns.

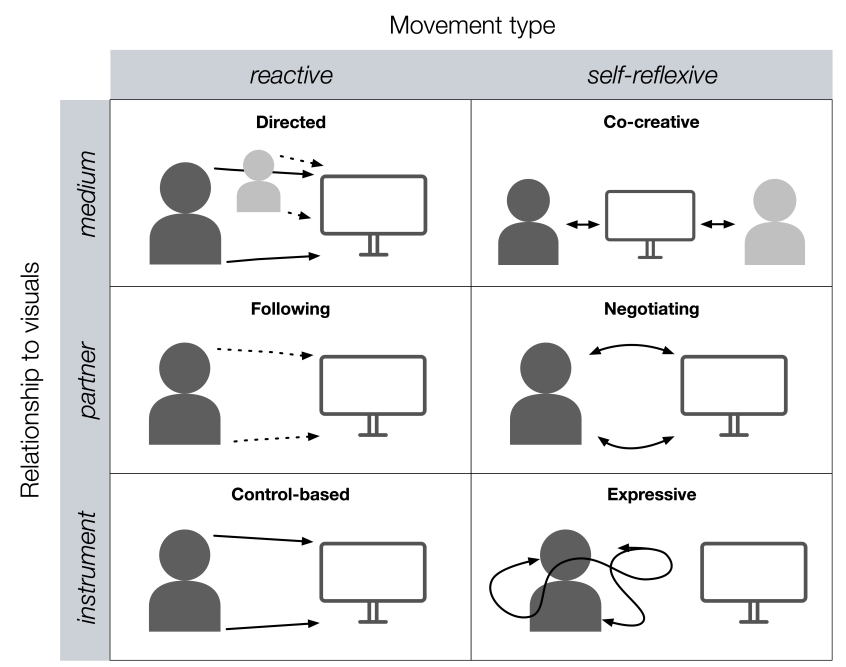

Figure 2. A taxonomy of interaction patterns with our Kinect-based visualization system (the arrows represent user's direction of attention).

\section{Six modes of interaction}

Instrument/reactive: control-based interaction. A dancer can use the visualization system as an instrument with restrictive properties, resulting in movements that are aimed at understanding the visual mechanisms. In these scenarios, the dancer spends time figuring out the rule system of the visual environment in order to control its various elements.

The apparatus bound to the visuals, such as the Kinect, constrains the type of movement dancers are able to make. Dancers reconfigure their bodies in very specific ways to be sensed by the Kinect, such as moving within its field of view. For example, D3W1 talked about making bigger movements so that the Kinect can see them. In this way, the dancer becomes the "data source for Kinect" (CW3) and they spend time figuring out the points at which they "become invisible" to it. CW3 was highly aware of Kinect's presence in the studio and frequently directed the dancers to stay within its triangular space in order to be captured by the camera. She said: "I would spend a lot of time inside Kinect to learn its behaviors and where it fails". The probing movements that resulted were intended for controlling the apparatus.

Similarly, a dancer can explore her visualized body as an instrument, testing its limits. D2W3 for example remarked that she would spend a long time in front of the visuals, "Just moving one hand - does it have an effect? What about moving the fingers?" This cause-and-effect relationship one engages in with the visuals can lead to passive movements where dancers adapt to the limits of the visual environment. For example, in interacting with the "blobby form", D1W2 called two other dancers forward to join her in creating a "snail" shape on-screen with their combined bodies. These "postures-like" movements were decidedly different from movements she created when interacting with the "springs", which were more "dancerly" and used more space. D3W2 also controlled the blob to create an "L-shape" by lowering his body all the way to the ground, into a fetal position and raising his leg.

Instrument/self-reflexive: expressive interaction. When a dancer uses the visuals as an instrument to afford future movement possibilities, her focus turns from controlling the external visual behaviors back onto her body, resulting in expressive interaction with the visuals where the dancer embodies the visual behaviors, choreographing not the visuals themselves (as seen in "control-based" interaction) but rather her relationship to the visuals. In other words, the dancer no longer manipulates the behaviors of the visuals (such as the shape or speed of the springs); rather, she turns her attention to the manner in which a specific visual behavior can be attained (e.g. skipping like a feather to make the springs jump around). From the dancer's point of view, there is an increased focus on the bodily sensations of a movement. And from an observer's point of view, the dancer spends less time looking at the screen and more time moving in space.

D3W2, after understanding the behaviors of the spring system, began darting back and forth in space, making fluid turns, fully incorporating the elasticity of the springs into her movements. Interacting with the same spring system, D2W3 saw her virtual body as an extended body: "I don't have any shoulder or hips: [they] are morphed into the body". Absorbing this quality into her physical body and using it as a motif to inspire movements, she built movement sequences around extending motions of her arms and legs in response to the "continuous" and "structureless" nature of the springs.

Partner/reactive: "following" interaction. Instead of being an extension to the dancer's body, the virtual body can also be seen as a disembodied image, or a partner, that constrains actions. Here the dancer produces movements that are courteous or polite toward the virtual partner. She waits around, letting the visuals breathe and finish their movements, or she follows the movements of the virtual partner.

D1W2, in interacting with the springs, moved her arms in a wave-like way, imitating the type of motion the springs were making. When interacting with the "trails", she moved her fingers to correspond to the rate at which the trace moved, even though the finger movements were not seen by the camera nor were they visualized on-screen. D4W2, during interaction with the springs, made skipping movements to match the buoyant motions on the screen. Similarly, D5W2, 
while moving to the "particles", raised her shoulders when the particles were flying upwards, lifting her entire body upward to match the flying motion of the particles. D1W2 also matched her movement speed to the speed of the moving particles.

In addition to following the visuals' movements, dancers also spent time letting the visuals play out their own movements. D2W2 moved less when she was interacting with the particles, in comparison to other visualizations, because the particles had a lot of movement themselves. Similarly, D2W3 stayed in front of the camera after she made a very tiny movement with her hand, watching the springs move on their own.

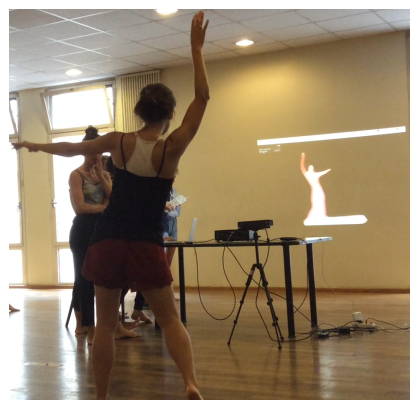

(a)

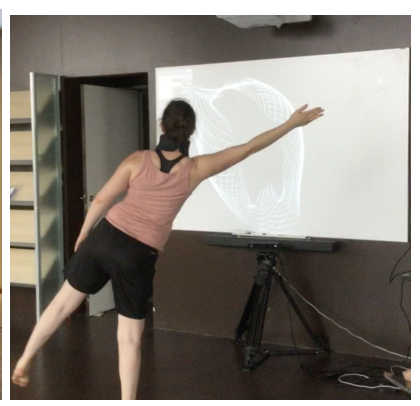

(b)
Figure 3. Dancers interacting with the visuals in (a) Workshop 2 (b) Workshop 3

Partner/self-reflexive: "negotiating" interaction. Dancers can also take a more active role, in relation to their virtual partner, in creating movements that are self-initiated rather than imitative. For example, D5W2 used almost the same movement sequence throughout all of her interactions with the visuals (she also appeared in Workshop 1 with an earlier version of the probe). Instead of making up movements on the spot and changing them according to each visualization, she would stick to her movement sequence, catching a glance every now and then at the visuals. She appeared to push back at her visualized body: when the particles were fading away and their speed very slow, instead of following the motion, she made faster movement with her arms, seemingly trying to get the visuals to come back to life. D4W2 also exhibited this negotiating behavior in interacting with the spring system. When the rigidity of the spring increased, he made a motion to grab a hold of an invisible body in space and tried to move it slowly around. When the rigidity of the spring was relaxed (i.e. more springiness back), he started to take the invisible body and thrash it around vigorously.

Medium/reactive: directed interaction. The visuals were also used as an interface for dancer-to-dancer communication.
The students from Workshop 1 choreographed each other based on the visual effects the movements created. They told each other the specific spots in space to move to or emphasized a particular gesture in order to create a visual effect. The visuals allowed them to see the effects of their movements, turning something internal into an object with which everybody can see and interact. Students also exchanged movement ideas via the visuals. D6 from the same workshop saw her colleague make a brush effect with the trails by swinging her arms. She liked how it looked visually and tried to recreate the same effect by moving her whole body across the space.

Medium/self-reflexive: co-creative interaction. Towards the end of Workshop 3, the choreographer manipulated the parameters on the visualization the dancer was interacting with. We observed a moment where she changed the parameters so that visual movement on-screen reflected the quality of movement the dancer was executing at the time. The choreographer also made choreographic suggestions via visuals a burst of energy in the springs by rapidly increasing and decreasing the "springiness" parameter. In response, the dancer adapted her movements, making a swift turn and a swish of the arm. In this way, the choreographer and the dancer became entangled in a duet via the visualization. Here, the visuals become a medium through which the choreographer and the dancer communicate.

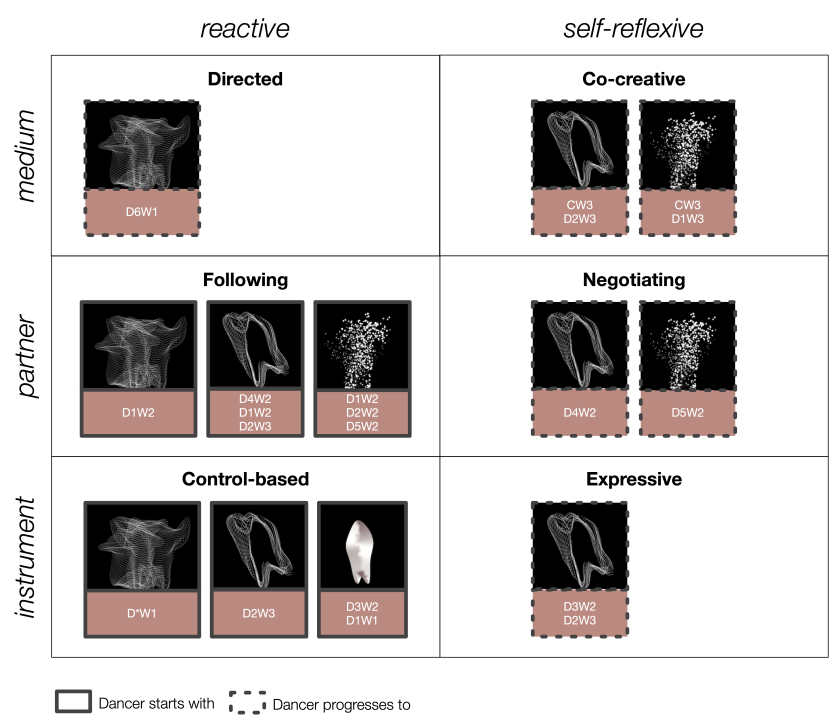

Figure 4. The participants' interaction patterns with each visualization.

We relate the "instrument" relationship dancers form with the visuals to "toolness", or the extent to which the visuals can be used as a tool to reflect body form or movement structure. Trails, blob, and springs all score high here because 
they allow the dancers to make shapes with their bodies or movements in space. Referring to Figure 4, we can see all three of them were used as instruments in workshops. Next, we relate the "partner" relationship to "agency", or the extent to which the visuals exhibit unanticipated movement behaviors or autonomy. Both the particles and the springs have a high amount of self-movement in response to user movements, and as a result, they were often viewed as interactive partners rather than instruments with which to perform operations. Finally, the "configurability" of the visuals contributes to the extent to which they can mediate human-human communication. Both particles and springs have many parameters to manipulate, making them good candidates for the dancer-choreographer duet we saw above.

\section{Strategies for inventing new movements}

As we can see in Figure 4, dancers can enter into a variety of interaction modes with any of the visualizations. While we resist quantifying our data so as to indicate absolute trends, we wish to note that many of our participants enter into "control-based" or "following" patterns when first interacting with the visuals. Over time, they develop strategies that allow them to move into other types of interactions, which we detail below.

Construction of complex relationships. The visuals are objects filled with significance and potential for action. That potential is released via meaning-making: creating complex relationships that go beyond the original cause-and-effect relationship. This strategy enables the dancer to switch from "control-based" and "following" interactions to "negotiating".

D1W3 struggled in the beginning to interact with the visuals. Trained in modern jazz, she does not have extensive experience in improvisation. She was unable to make a connection to the visuals and come up with movement sequences that were not simply controlling gestures. The choreographer stepped in and helped guide her in relating to the visuals: "Explore following [the particles] continuously in space. [You can follow] with the entire body or with different body parts...forward or backward in space". The dancer noticed the change in her movements, saying, "When I stopped looking at the effects on-screen, I started to make interesting movements. [The visualizaion] can sometimes trap you - it's hard to go beyond its limits". In this case, D1W3 was able to relate to the visuals that were more complex than the original movement to visual mappings designed. PW3 observed that the meaning-making process "produces something that is less of a direct mapping from [visual] effect to [movement] shape, but a more complex mapping" because of the way visual motion on-screen "is decoupled from the movement of the person in space".
D2W3 perceived the spring-like figures on-screen as butterflies surrounding her virtual body. As soon as she made that connection, she was no longer tethered to the visuals by the original mappings where springiness corresponded to the speed of user movement. She began to play more with the physical space, imagining butterflies filling the 3D space around her. By creating these complex relationships to the visuals, she was able to engage in the dynamic interplay between the sensual and spatial dimensions in relation to the visuals.

Progressive learning of system behaviors. Dancers can shift from "control-based" mode to "expressive" or "negotiating" by switching modality from visual to kinaesthetic. This is characterized by progressive learning of system behaviors.

We observed that when dancers use the visuals as an instrument, they progressively absorb the instrument into their own bodies and hence adapting their own body schema in the process. This can deform the dancer's perception of the physical space and was felt by dancers from all workshops. D2W3 expressed, "We don't know body boundaries because of the visualizations' virtual space.". D4W2 also said, "I had a mental map [of my spatial relationship] with regards to others, but when I look at the falling particles - which were atmospheric, abstract, and geometric - I could not enter into this [map]". CW3 also commented, "When you put the dancers in front of the Kinect, it changes their relationship to space and their dimensionality of their body is very flattening". D1W1 echoed that she felt she was moving along "the plane" when interacting with the visuals.

In absorbing the tool, dancers reconcile the physical studio space with the virtual one. Once the instrument and the space configuration that comes with each instrument is absorbed into the body, dancers are able to take back their agency and be in control of the instrument, without completely surrendering to its will. This process of transforming the body to fit the constraints of the visuals can be seen as a type of skill acquisition [52].

Skill acquisition also occurs when dancers learn and embody the visual behaviors over time. D1W3 saw the spring movements as "spaghetti-like". As she became acquainted with this behavior, she started to incorporate it into her own physical movements without looking at the screen, exploring the bodily sensations corresponding to the malleability of the "spaghetti springs".

These examples demonstrate the correlation between learning and creative uses of the visuals. One obvious strategy to perturb dancers' natural tendency would be to vary the visuals repeatedly so as to prevent the dancers from forming relationships with them and thus falling into specific patterns of interactions. However, if the transition between defamiliarization and learning is not handled well, it might 
lead to the user feeling a loss of agency or the negative impacts of a constraint, leading to movements that are passive rather than self-initiated and exploratory. D1W2 remarked, regarding the frequent changing of visual parameters by one colleague, "the changing makes the interaction difficult", adding, "Sometimes I feel it's the manipulator who is varying the parameters; other times I feel it's me who is influencing [the visuals]". CW3 said, "I would not change the parameters too often because I think it takes the agency away from the dancers to learn new relationships". For dancers to respond flexibly to changing visuals while maintaining engagement and control, attention should be paid to facilitating the learning process.

Mediated movement exploration. Creative uses of the visuals can also take place via the technology-mediated duet we saw in the previous example between the dancer and the choreographer, facilitating transition into "co-creative" interaction. The duet is made possible by the externalization of creative impulses on the part of the choreographer and the dancer. The dancer who took part in that duet commented, "In [traditional] improvisation, [the process is] usually internal. There's something external here. People can guide you to discover movements.". When D1W3 was moving without facing the screen, CW3 used the visuals to guide her movements, reminding her of the particles' behaviors - "The particles have a delayed response in reaction to you" - or of the camera's point of view - "When you're close to the front [of the camera], there's a lot of [particle] movement." With that, the dancer could respond by stretching her movement and moving back in space without breaking her movement flow.

\section{DISCUSSION}

We have shown above that dancers' bodies contain intelligence to support smooth transitions between complex changes of bodily space, creative expressions, and social relations. Rather than taking a technological approach, attempting to analyze, isolated from real practices, the properties or reasons an artifact can cause a specific effect, we adopt a practice-lens approach, championed by Orlikowski [43], to study how these dancers innovate within the confines of a given technology. This allows us to identify six types of technology use and the specific ways in which dancers navigate between different uses. This serves as a rich account for how kinaesthetic creativity takes place with an interactive artifact. From this view, creativity, and consequently, the ability to imagine new possible futures, emerges out of active exploration and engagement of all participants in the studio space: the dancers, the choreographer, and the visuals.

\section{Relationships to visuals}

We found that dancers form different relationships with the visuals. When used as an instrument, the visuals extend the boundaries of dancers' body to include external devices such as the Kinect. Once these artifacts become incorporated into the body, they become part of the "structure of the lived body" [52]. With that comes an adapted space for movement. When the dancer from Workshop 3 remarked that her phsyical space felt "deformed" during interaction with the visuals, she was feeling the process of her body adapting to a newly constructed space that includes the Kinect's range of capture and the visuals' virtual space.

The visuals, by virtue of representing dancers' movements on-screen, help dancers form a "partner" relationship with them. We can see this as an example of "externalization" studied by Kirsh [29]. Kirsh uses Willliam Forsythe's Improvisation Technologies ${ }^{4}$ to illustrate how internal choreographic thought can be reified into a visible object with which he then uses to communicate movement ideas such as "shearing" and "torsion". In our examples, the visualizations similarly serve as reification of movement ideas, allowing dancers to see the shape, structure, and dynamics of their movements. In addition to using these visualized "objects of thought" as things to manipulate and to think with, dancers negotiate with them like a partner who can push back and make suggestions.

Finally, the visualizations also serve to mediate the relationship between people in the studio space. In What Things Do [54], Verbeek gave an example of the dinner table mediating the social interactions of the people sitting around it delineating how close people can sit next to one another. In a similar fashion, the visualizations mediate the relationships between the dancers or between dancer and choreographer by configuring how each person can control the visual elements. Just as changing the table settings can change social relationships, changing the visuals can change how people in the studio space relate to one another, thereby establishing an implicit visual communication channel over which movement intentions can be expressed.

\section{Movement types}

In our study, dancers generate two types of movements: reactive and self-reflexive. From the interaction point of view, reactive movements are related to Heidegger's "present-athand" mode of engagement and self-reflexive movements are related to "ready-to-hand" [20]. Heidegger characterizes "present-at-hand" as objective reflection. In "reactive movements", dancers probed around each visualization in order to understand its potential for action. This is different from "self-reflexive movements", where dancers fully embody the visual behaviors, shifting their focus out of the utilities of

\footnotetext{
${ }^{4} \mathrm{~A}$ series of visually anootated videos featuring Forsythe forming and transforming various geometric shapes with his body: http://www.niea.unsw.edu.au/research/publications/improvisationtechnologies-tool-analytical-dance-eye
} 
the visuals and towards the body. The visuals, by fading into the background, become "ready-to-hand". From the user's perspective, dancers adapt to the affordances of the visuals during reactive movements and appropriate the visuals to suit their own practices during self-reflexive movements.

\section{ANALYSIS OF EXISTING MOVEMENT-BASED SYSTEMS}

We turn to place the interaction patterns we observed within the larger context of movement-based systems in the arts and HCI in order to see whether similar behaviors can be found. This will show the generalizability of these patterns and the utility of the two dimensions in classifying movement-based systems. We stress that this shows predominant trends of particular systems. This does not suggest that the systems ossify specific forms of interaction.

Control-based: systems for gestural control: Existing systems that fall under the instrument/reactive category often require the user to learn a tool and use it to perform a specified goal. An example is Light Music by Thierry de $\mathrm{Mey}^{5}$, which is a contemporary musical piece where a gestural instrument is employed: the musician manipulates sound events by making percussive gestures. Connected by Chunky Move $^{6}$ is a dance piece where the dancers are tethered to a kinetic sculpture, using their choreographed movements to control it. There are also many examples in gaming where the player uses their body as a controller to reach a goal, such as playing tennis with Wii. In these systems, user's attention is often on the consequences of their movements on the external world [50], be it sonic, physical, or visual.

Expressive: systems for movement exploration: In our study, the dancers were seen embodying visual behaviors during expressive interaction. In existing literature, there is a rich set of systems and environments that supports expressive movement exploration via carefully designed affordances that allow for surprises and discovery. These systems are different from those in the previous category because movements here, instead of targeting external digital events, use feedback from these events to reflect on movements themselves. Systems like hipDisk [56] and Still, Moving [16] sonify user movements to promote explorations of hip motions or micro-movements in stillness.

Following: systems for movement learning: "Following" interactions can be found in systems designed to be partners in supporting the user reach a goal, such as XBox's Just Dance ${ }^{7}$. The user copies the virtual partner's movements and get feedback on how well they are executing those movements. The augmented climbing wall [28], which guides the

\footnotetext{
${ }^{5}$ http://brahms.ircam.fr/works/work/22063/

${ }^{6}$ http://www.danceaustralia.com.au/review/chunky-move-connected

${ }^{7}$ https://www.microsoft.com/en-us/p/just-dance-2018/
}

user through a series of tasks via projected feedback, also falls under this category.

Negotiating: systems with embedded AI: Existing systems supporting a partner relationship and self-reflexive movements are often characterized by integration of artificial intelligence, allowing the artificial agent to interact with the user in creative ways. An example that falls under this category is the Choreographic Language Agent ${ }^{8}$ that lets the user define a set of rules which will be used by the agent to generate movements of its own. This agent can suggest movements for the dancer to consider during improvisation. Another example is Viewpoints AI [27], where a projected artificial agent responds to and adapts to a human interactor.

Directed: systems for co-located play: Systems that are used as a medium to produce reactive movements often question the boundaries of control between two human users via technologically-mediated interaction. Examples can be found in systems for co-located play such as Balance Ninja [3], where two players stand on a balance beam trying to throw the other off-balance while staying on themselves. As one player leans, the muscle stimulation system worn by both players will induce lean on the other player. In this case, each player's movements are the result of reacting to and pushing back on the other player. ACCESS $^{9}$ is an interactive installation where a web user can track anonymous individuals in a public space by pursuing them with a projected spotlight. The dodging movements that result on the part of the tracked individuals are reactive movements; the interaction between the "tracker" and the "trackee" are mediated by the robotic beam and the underlying tracking system.

Co-creative: systems for social interaction: Finally, systems promoting co-creativity are characterized by their ability to mediate human-to-human interactions, revealing movement characteristics that become resources for creative expression. One example is Boundary Functions by Scott Snibbe ${ }^{10}$, which is an interactive installation that visualizes the dynamic spatial relationships between participants. Each time a participant steps into the installation space, projected lines on the floor adapt themselves to reflect their personal space in relation to others. Participants then explore the changing personal spaces by walking around the installation. The projection mediates how one relates to another spatially.

We note that although this does not comprise an exhaustive set, the taxonomy serves to contextualize the interaction patterns we observed and illuminate that they can be found in movement-based interaction in the arts and HCI. Most notably, this framework provides an anlytic lens through

\footnotetext{
${ }^{8}$ http://waynemcgregor.com/research/choreographic-language-agent ${ }^{9}$ http://www.sester.net/access/

${ }^{10}$ https://www.snibbe.com/projects/interactive/boundaryfunctions/
} 
which to look at existing systems and to draw out subtle nuances between them.

\section{IMPLICATIONS FOR DESIGN}

As we observed in our study, dancer's ability to transition between different modes of interaction with the visuals is driven by various strategies they employ to invent new movements. We propose that by explicitly designing for this shift, we enable users to enter into active dialogue with the system, allowing them to engage with creative possibilities via exploration, negotiation, and mediation. We see indeterminacy, discoverability, appropriability, and correspondence as qualities that invite interweaving perspectives, and thus estrangement, which we argue characterizes kinaesthetic creativity.

\section{Leveraging indeterminacy to facilitate constructing complex relationships}

The visuals we implemented are all open for interpretation to varying degrees. Dancers used this indeterminacy to construct meaningful relationships to the visuals, allowing them to move beyond the original mappings. The notion of "openness for interpretation" is articulated by Chalmers and Gaver et al., who advocate for systems that can be "flexibly interpreted" [6] and that encompass the "many and nuanced ways people organize and give meaning to their activities" [18].

Both Text Rain ${ }^{11}$ and Videoplace ${ }^{12}$ are systems that support open exploration by leveraging indeterminacy in the system. They allow users to make different possible connections with the visuals that are personally meaningful to them, either by holding up the falling texts with their arms or picking up shapes by forming a circle with their hands. The systems, though inexplicit in the specific gestures to use, are clear in their grammars of interaction, or system-defined goals (e.g. picking things up with body parts). Consequently, users can create personalized gestures, embedding symbolic meaning in their interactions spontaneously and in a situated manner. This is similar to what Simondon refers to as the "margin of indetermination" [48] of objects. He argues that technical objects should maintain a margin indetermination, and it is via this margin that humans are able to form meaningful interrelationship with the machines.

\section{Employing discoverability to guide progressive learning}

As we observed in our study, too many changing visual elements in the beginning of interaction can break dancers' sense of agency, resulting in their inability to form meaningful relationship to the visuals, further inhibiting their

\footnotetext{
${ }^{11}$ by Camille Utterback: http://camilleutterback.com/projects/text-rain/

${ }^{12}$ by Myron Krueger: http://www.medienkunstnetz.de/works/videoplace/
}

creative expression of movement. Therefore, achieving expertise and constructing complex relationships are closely linked. Without support to learn and discover an interface, meanings cannot be properly formed. For this learning to take place, systems should make their technological inner workings discoverable $[9,51]$.

We apply the notion of discoverability to movement improvisation with an AI system. In our workshops, dancers were able to make self-initiated exploratory movements after figuring out the constraints of the setup, i.e. how the system "sees" them (e.g. Kinect's field of view or the mapping rules for translating movement data to visuals). Similarly, an AI system, by making visible and manipulatable the underlying logic it uses to construct an understanding of the users, can support them in transitioning from passive to active interactions with it. This gives users control over how they wish to be perceived, further helping them understand and anticipate the system responses. This raises subsequent design-related questions: What are the different ways of representing this logic? What is the appropriate level of intelligibility?

\section{Considering appropriability and correspondence to enrich movement exploration}

As users progress from novices to experts, they may wish to evolve to more varied or powerful interaction with the system. During the workshops, mediated communication via visuals was a way for dancers to try out suggestions made by others and explore movement ideas that may not otherwise occur to them. The configurability of the visualization parameters further allowed for transmission of more complex ideas, materialized in visual movements. This opens up opportunities to reflect on the notion of "appropriability" [8] as a method for exploring alternative paths to a system's pre-designed mechanisms. If these "inner workings", once made discoverable, can be reassembled, reconfigured, and reappropriated, the user can more readily improvise with them, leading to serendipitous discoveries.

The improvisatory dialogues during movement making in our workshops can be conceptualized as a practice of correspondence [26], which refers to the circulatory flow between the maker and their materials, where, rather than applying an already existing form to shapeless matter (a "hylomorphic" approach), the maker is attuned to the forces of the materials and grows with them in making. Movements produced in our workshops are interesting materials to consider through this lens because, unlike clay or textile, they do not exist outside of the maker - they are bound up in the dancer's body, where the movement traces are often felt rather than seen, effectively resisting a "hylomorphic" treatment with their transience. Extending this view, it can be interesting for choreographic tools to prevent these movement traces from falling through the cracks during their becoming, and to 
explore ways beyond just capturing and representing these traces (e.g. with videos), but of making them interactive in order to better engage with their generative potential.

\section{CONCLUSION}

Our study sheds light on the various ways dancers interact with visuals, which we used to probe the dancers' process in generating new movement material, or what Svanaes refers to as kinaesthetic creativity: the body's ability "to explore possible futures in a creative manner" [52]. We found that dancers' kinaesthetic creativity is driven by their ability to shift between different interaction patterns via constructing complex relationships, progressive learning, and mediated movement exploration. By employing a technology probe approach to understanding how dancers explore movement alternatives, we are able to gain valuable insights into processes that are otherwise complex, layered, and hard to unpack. To support this generative flux of dynamic relations and forces, technologies should mediate flexible oscillations between different modes of interaction - by designing for indeterminacy, discoverability, appropriability, and correspondence.

\section{ACKNOWLEDGMENTS}

We thank the participants for their time and insights. We also thank the reviewers whose feedback have improved this work. This work was partially supported by European Research Council (ERC) grant № 321135 “CREATIV: Creating Co-Adaptive Human-Computer Partnerships”.

\section{REFERENCES}

[1] Susanne Bødker. 2006. When second wave HCI meets third wave challenges. In Proceedings of the 4th Nordic conference on Human-computer interaction: changing roles. ACM, 1-8.

[2] Virginia Braun and Victoria Clarke. 2006. Using thematic analysis in psychology. Qualitative research in psychology 3, 2 (2006), 77-101.

[3] Richard Byrne, Joe Marshall, and Florian'Floyd' Mueller. 2016. Balance ninja: towards the design of digital vertigo games via galvanic vestibular stimulation. In Proceedings of the 2016 Annual Symposium on Computer-Human Interaction in Play. ACM, 159-170.

[4] Diogo Cabral, Urândia Carvalho, João Silva, João Valente, Carla Fernandes, and Nuno Correia. 2011. Multimodal video annotation for contemporary dance creation. In CHI'11 Extended Abstracts on Human Factors in Computing Systems. ACM, 2293-2298.

[5] Kristin Carlson, Thecla Schiphorst, and Philippe Pasquier. 2011. Scuddle: Generating Movement Catalysts for Computer-Aided Choreography.. In ICCC. 123-128.

[6] Matthew Chalmers. 2004. A historical view of context. Computer Supported Cooperative Work (CSCW) 13, 3-4 (2004), 223-247.

[7] Marianela Ciolfi Felice, Sarah Fdili Alaoui, and Wendy E. Mackay. 2018 Knotation: Exploring and Documenting Choreographic Processes. In Proceedings of the 2018 CHI Conference on Human Factors in Computing Systems (CHI '18). ACM, New York, NY, USA, Article 448, 12 pages. https://doi.org/10.1145/3173574.3174022

[8] Alan Dix. 2007. Designing for appropriation. In Proceedings of the 21st British HCI Group Annual Conference on People and Computers: HCI... but not as we know it-Volume 2. BCS Learning \& Development Ltd., 27-30.

[9] Paul Dourish. 2004. Where the action is: the foundations of embodied interaction. MIT press.

[10] Universal Everything. 2018. Website. http://universaleverything.com/

[11] Sarah Fdili Alaoui, Frederic Bevilacqua, Bertha Bermudez, and Christian Jacquemin. 2013. Dance Interaction with physical model visualization based on movement qualities. International fournal of Arts and Technology, IfART (2013), 0-12.

[12] Sarah Fdili Alaoui, Baptiste Caramiaux, Marcos Serrano, and Frédéric Bevilacqua. 2012. Movement qualities as interaction modality. In Proceedings of the ACM SIGCHI Conference on Designing Interactive Systems (DIS'12). ACM Press, Newcastle, UK, 761-769.

[13] Sarah Fdili Alaoui, Jules Françoise, Thecla Schiphorst, Karen Studd, and Bevilacqua, Frédéric. 2017. Seeing, Sensing and Recognizing Laban Movement Qualities. In In Proceedings of ACM Conference on Human Factors in Computing Systems (CHI). ACM Press.

[14] John C Flanagan. 1954. The critical incident technique. Psychological bulletin 51, 4 (1954), 327.

[15] Maiken Hillerup Fogtmann, Jonas Fritsch, and Karen Johanne Kortbek. 2008. Kinesthetic Interaction: Revealing the Bodily Potential in Interaction Design. In Proceedings of the 20th Australasian Conference on Computer-Human Interaction (OZCHI '08). ACM, Cairns, Australia, 89-96. https://doi.org/10.1145/1517744.1517770

[16] Jules Françoise, Yves Candau, Sarah Fdili Alaoui, and Thecla Schiphorst. 2017. Designing for Kinesthetic Awareness: Revealing User Experiences Through Second-Person Inquiry. In Proceedings of the 2017 CHI Conference on Human Factors in Computing Systems (CHI '17). ACM, New York, NY, USA, 5171-5183. https://doi.org/10.1145/3025453. 3025714

[17] Jules Françoise, Sarah Fdili Alaoui, Thecla Schiphorst, and Frédéric Bevilacqua. 2014. Vocalizing Dance Movement for Interactive Sonification of Laban Effort Factors. In Proceedings of the ACM SIGCHI Conference on Designing Interactive Systems (DIS '14). ACM, Vancouver, Canada, 1079-1082.

[18] William Gaver, Mark Blythe, Andy Boucher, Nadine Jarvis, John Bowers, and Peter Wright. 2010. The prayer companion: openness and specificity, materiality and spirituality. In Proceedings of the SIGCHI conference on Human factors in computing systems. ACM, 2055-2064.

[19] Lise Amy Hansen and Andrew Morrison. 2014. Materialising Movement-Designing for movement-based digital interaction. (2014).

[20] Martin Heidegger. 1962. Being and time (J. Macquarrie \& E. Robinson, trans.).

[21] Martin Heidegger. 1988. The basic problems of phenomenology. Vol. 478. Indiana University Press.

[22] Kristina Höök, Martin P Jonsson, Anna Ståhl, and Johanna Mercurio. 2016. Somaesthetic appreciation design. In Proceedings of the $2016 \mathrm{CHI}$ Conference on Human Factors in Computing Systems. ACM, 3131-3142.

[23] Caroline Hummels, Kees C Overbeeke, and Sietske Klooster. 2007. Move to get moved: a search for methods, tools and knowledge to design for expressive and rich movement-based interaction. Personal and Ubiquitous Computing 11, 8 (2007), 677-690.

[24] Caroline Hummels and Jelle Van Dijk. 2015. Seven principles to design for embodied sensemaking. In Proceedings of the Ninth International Conference on Tangible, Embedded, and Embodied Interaction. ACM, 21-28.

[25] Hilary Hutchinson, Wendy Mackay, Bo Westerlund, Benjamin B Bederson, Allison Druin, Catherine Plaisant, Michel Beaudouin-Lafon, Stéphane Conversy, Helen Evans, Heiko Hansen, et al. 2003. Technology probes: inspiring design for and with families. In Proceedings of the SIGCHI conference on Human factors in computing systems. ACM, 17-24. 
[26] Tim Ingold. 2013. Making: Anthropology, archaeology, art and architecture. Routledge.

[27] Mikhail Jacob and Brian Magerko. 2015. Viewpoints ai. In Proceedings of the 2015 ACM SIGCHI Conference on Creativity and Cognition. ACM, 361-362.

[28] Raine Kajastila and Perttu Hämäläinen. 2014. Augmented climbing: interacting with projected graphics on a climbing wall. In CHI'14 Extended Abstracts on Human Factors in Computing Systems. ACM, 1279-1284.

[29] David Kirsh. 2010. Thinking with external representations. Ai \& Society 25, 4 (2010), 441-454.

[30] Golan Levin. 2018. Website. http://www.flong.com/

[31] Aaron Levisohn and Thecla Schiphorst. 2011. Embodied engagement: Supporting movement awareness in ubiquitous computing systems. Ubiquitous Learning: An International fournal 3 (2011), 97-111.

[32] Lian Loke, George Poonkhin Khut, Maggie Slattery, Catherine Truman, Lizzie Muller, and Jonathan Duckworth. 2013. Re-sensitising the body: interactive art and the Feldenkrais method. International fournal of Arts and Technology 6, 4 (2013), 339-356. https://doi.org/10.1504/ IJART.2013.058283

[33] Lian Loke and Toni Robertson. 2013. Moving and making strange: An embodied approach to movement-based interaction design. $A C M$ Transactions on Computer-Human Interaction (TOCHI) 20, 1 (2013), 7.

[34] Pedro Lopes, Alexandra Ion, Willi Mueller, Daniel Hoffmann, Patrik Jonell, and Patrick Baudisch. 2015. Proprioceptive Interaction. In Proceedings of the 33rd Annual ACM Conference on Human Factors in Computing Systems (CHI '15). ACM, New York, NY, USA, 939-948. https://doi.org/10.1145/2702123.2702461

[35] Diego Silang Maranan, Sarah Fdili Alaoui, Thecla Schiphorst, Philippe Pasquier, and Lyn Bartram. 2014. Designing For Movement : Evaluating Computational Models using LMA Effort Qualities. In Proceedings of the 2014 CHI Conference on Human Factors in Computing Systems (CHI'14). ACM, Toronto, Canada.

[36] Elena Márquez Segura, Laia Turmo Vidal, Asreen Rostami, and Annika Waern. 2016. Embodied Sketching. In Proceedings of the 2016 CHI Conference on Human Factors in Computing Systems (CHI '16). ACM, San Jose, CA, USA, 6014-6027.

[37] Helena Mentis and Carolina Johansson. 2013. Seeing Movement Qualities. In Proceedings of the $2013 \mathrm{CHI}$ Conference on Human Factors in Computing Systems (CHI'13). ACM, Paris, France, 3375-3384.

[38] Jin Moen. 2007. From hand-held to body-worn: embodied experiences of the design and use of a wearable movement-based interaction concept. In Proceedings of the International Conference on Tangible, Embedded, and Embodied Interaction (TEI'07). ACM, 251-258.

[39] Jin Moen and Johan Sandsjö. 2009. BodyBug-Design of KinAesthetic Interaction. Nordes 1 (2009).
[40] Florian'Floyd' Mueller, Richard Byrne, Josh Andres, and Rakesh Patibanda. 2018. Experiencing the Body as Play. In Proceedings of the 2018 CHI Conference on Human Factors in Computing Systems. ACM, 210.

[41] onformative. 2018. Website. https://www.onformative.com/

[42] OpenEndedGroup. 2018. Website. http://openendedgroup.com/

[43] Wanda J Orlikowski. 2008. Using technology and constituting structures: A practice lens for studying technology in organizations. In Resources, co-evolution and artifacts. Springer, 255-305.

[44] Thomas Ots. 1994. The silenced body-the expressive Leib: on the dialectic of mind and life in Chinese cathartic healing. Embodiment and experience: The existential ground of culture and self 116 (1994).

[45] Thecla Schiphorst. 2011. Self-evidence: Applying Somatic Connoisseurship to Experience Design. In CHI '11 Extended Abstracts on Human Factors in Computing Systems (CHI EA '11). ACM, New York, NY, USA, 145-160. https://doi.org/10.1145/1979742.1979640

[46] Maxine Sheets-Johnstone. 2011. The primacy of movement. Vol. 82. John Benjamins Publishing.

[47] Richard Shusterman. 2008. Body Consciousness: A Philosophy of Mindfulness and Somaesthetics. (01 2008), 1-239.

[48] Gilbert Simondon. 1958. Du mode d'existence des objets techniques. (1958).

[49] Scott Snibbe. 2018. Website. https://www.snibbe.com/

[50] Noé Soulier. 2016. Actions, mouvements et gestes. Centre national de la danse.

[51] Lucy Suchman. 2007. Human-machine reconfigurations: Plans and situated actions. Cambridge University Press.

[52] Dag Svanæs. 2013. Interaction design for and with the lived body: Some implications of merleau-ponty's phenomenology. ACM Transactions on Computer-Human Interaction (TOCHI) 20, 1 (2013), 8.

[53] Camille Utterback. 2018. Website. http://camilleutterback.com/

[54] Peter-Paul Verbeek. 2005. What things do: Philosophical reflections on technology, agency, and design. Penn State Press.

[55] Rudolf von Laban. 1975. Modern educational dance. Princeton Book Co Pub.

[56] Danielle Wilde. 2012. hipDisk: experiencing the value of ungainly, embodied, performative, fun. In CHI'12 Extended Abstracts on Human Factors in Computing Systems. ACM, 979-982.

[57] Danielle Wilde, Thecla Schiphorst, and Sietske Klooster. 2011. Move to design/design to move: a conversation about designing for the body. interactions 18, 4 (2011), 22-27.

[58] Danielle Wilde, Anna Vallgårda, and Oscar Tomico. 2017. Embodied Design Ideation Methods: Analysing the Power of Estrangement. In Proceedings of the 2017 CHI Conference on Human Factors in Computing Systems (CHI '17). ACM, New York, NY, USA, 5158-5170. https://doi. org $/ 10.1145 / 3025453.3025873$ 